\title{
Intraoperative fluid optimization using stroke volume variation in high risk surgical patients: results of prospective randomized study
}

\author{
Jan Benes*, Ivan Chytra, Pavel Altmann, Marek Hluchy, Eduard Kasal, Roman Svitak, Richard Pradl and Martin Stepan
}

\begin{abstract}
Introduction: Stroke volume variation (SVV) is a good and easily obtainable predictor of fluid responsiveness, which can be used to guide fluid therapy in mechanically ventilated patients. During major abdominal surgery, inappropriate fluid management may result in occult organ hypoperfusion or fluid overload in patients with compromised cardiovascular reserves and thus increase postoperative morbidity. The aim of our study was to evaluate the influence of SVV guided fluid optimization on organ functions and postoperative morbidity in high risk patients undergoing major abdominal surgery.
\end{abstract}

Methods: Patients undergoing elective intraabdominal surgery were randomly assigned to a Control group $(n=60)$ with routine intraoperative care and a Vigileo group $(n=60)$, where fluid management was guided by SW (Vigileo/ FloTrac system). The aim was to maintain the SWV below $10 \%$ using colloid boluses of $3 \mathrm{ml} / \mathrm{kg}$. The laboratory parameters of organ hypoperfusion in perioperative period, the number of infectious and organ complications on day 30 after the operation, and the hospital and ICU length of stay and mortality were evaluated. The local ethics committee approved the study.

Results: The patients in the Vigileo group received more colloid (1425 ml [1000-1500] vs. $1000 \mathrm{ml}$ [540-1250]; $P=$ 0.0028 ) intraoperatively and a lower number of hypotensive events were observed (2[1-2] Vigileo vs. 3.5[2-6] in Control; $P=0.0001)$. Lactate levels at the end of surgery were lower in Vigileo $(1.78 \pm 0.83 \mathrm{mmol} / \mathrm{l} \mathrm{vs} .2 .25 \pm 1.12 \mathrm{mmol} / \mathrm{l} ; P=$ 0.0252). Fewer Vigileo patients developed complications (18 (30\%) vs. 35 (58.3\%) patients; $P=0.0033$ ) and the overall number of complications was also reduced (34 vs. 77 complications in Vigileo and Control respectively; $P=0.0066$ ). A difference in hospital length of stay was found only in per protocol analysis of patients receiving optimization (9 [8-12] vs. 10 [8-19] days; $P=0.0421$ ). No difference in mortality (1 (1.7\%) vs. $2(3.3 \%) ; P=1.0)$ and ICU length of stay (3 [2-5] vs. $3[0.5-5] ; P=0.789)$ was found.

Conclusions: In this study, fluid optimization guided by SWV during major abdominal surgery is associated with better intraoperative hemodynamic stability, decrease in serum lactate at the end of surgery and lower incidence of postoperative organ complications.

Trial registration: Current Controlled Trials ISRCTN95085011.

\section{Introduction}

Fluid administration in the intraoperative period is an integral part of everyday anesthesiology practice. Adequate intravascular volume replacement is a crucial issue that can seriously affect postoperative organ function and

* Correspondence: benesj@fnplzen.cz

Department of Anesthesiology and Intensive Care, Charles University teaching hospital, alej Svobody 80, Plzen, 304 60, Czech Republic

Full list of author information is available at the end of the article hence outcome [1-3]. Guiding fluid management using standard physiologic variables (blood pressure, heart rate etc) may be associated with a state of occult hypoperfusion [4]. Goal-directed therapy (GDT) was proposed to overcome this problem by introducing different hemodynamic variables into a dynamic perspective of individualized fluid loading and use vasoactive substances to reach predefined goal of optimal preload and/or oxygen delivery $[5,6]$. 
In past years, many trials using different devices and goals have been published in the literature demonstrating better outcomes in organ functions $[7,8]$, morbidity [914] or even mortality [15]. Esophageal Doppler was used by many for guiding fluid management with good results but its use is partially limited by the need for deep sedation [16] and experienced staff [17]. Also, the reliability in major vascular procedures requiring cross-clamping of descendent aorta could be questioned. Different algorithms for arterial pressure wave analysis have been introduced lately. As arterial cannulation is routinely used for continuous blood pressure monitoring in high-risk patients, their use is not associated with increased invasivity and risk. These monitors are generally well tolerated by patients and easy to maintain. Some of these devices have already been used in GDT trials [12].

With the introduction of arterial pressure waveform analysis, the well-known interaction between stroke volume variation (SVV) and lung inflation during mechanical ventilation [18] has become available for routine clinical use. Several studies documented the usefulness of blood pressure variations and it surrogates (pulse pressure variation or systolic pressure variation) in predicting position on the Frank-Starling curve and hence fluid responsiveness [19-22]. The reliability of automated assessment [23], the influence of ventilator setting $[24,25]$ and afterload modification [26] were also addressed in the literature.

Vigileo/FloTrac is a continuous monitor of patient's hemodynamic status on a beat-to-beat basis using online analysis of arterial pressure waveform. Cardiac performance is calculated assessing the arterial tree impedance (defined as coefficient Khi - $\chi$ ), so no external calibration is needed and the device is ready to use after obtaining basic demographic parameters [27]. In past years, the arterial impedance calculation was criticized and use in clinical practice was debatable [28-30]. However, with software modifications and more frequent calculation of impedance, the device accuracy improved $[31,32,35]$. Despite some controversy $[36,37]$ it is used in clinical practice. SVV derived by Vigileo/FloTrac has shown good correlation with results acquired from the PiCCO system and with a cut-off value of $9.6 \%$ a good sensitivity and specificity for predicting fluid responsiveness [21]. The aim of our prospective randomized study was to examine the effect of SVV-guided fluid therapy in the perioperative care of high-risk surgical patients and its influence on postoperative morbidity and mortality in comparison with standard treatment.

\section{Materials and methods}

This was a prospective, randomized, partially blinded, single-center study conducted between July 2007 and May 2009 at the Department of Anesthesiology and
Intensive Care Medicine, at Charles University Teaching Hospital in Plzei. The trial was approved by local research ethics committee and all patients gave their informed consent. High-risk patients scheduled for major abdominal surgery with anticipated operation time longer than 120 minutes or presumed blood loss exceeding $1,000 \mathrm{ml}$ (i.e. colorectal or pancreatic resections, intraabdominal vascular surgery) were screened for eligibility. At least one operation-related and one patient-related inclusion criteria had to be fulfilled (Table 1). Patients younger than 18 years, with documented arrhythmias and with a weight below $55 \mathrm{~kg}$ or above $140 \mathrm{~kg}$ were excluded to ensure accuracy of Vigileo/FloTrac measurement [38].

\section{Patients randomization and outcome measures}

Patients meeting inclusion criteria were randomized using opaque sealed envelopes to intervention (Vigileo) or control (Control) group. The anesthetist responsible for intra-operative management was aware of the group assignment, whereas all other members of research team, other health care providers and patients were not. Randomization concealment for researchers was broken only at the end of the study for statistical data analysis. In case of intraoperative change in procedure performed (abandoning planned surgery because of inoperability or performing just a minor procedure), study protocol optimization was not realized, but their postoperative outcome was assessed in the intention-to-treat analysis (Figure 1)

Primary outcome measures were postoperative morbidity based on number of infectious and other organ

\section{Table 1: Inclusion criteria}

Procedure-related (at least one of them)

Operation duration more than 120 minutes and opened peritoneal cavity

Presumed blood loss more than 1,000 ml

\section{Patient related (at least one of them)}

Ischemic heart disease or severe heart dysfunction

Chronic obstructive pulmonary disease (moderate to severe)

Age above 70 years

ASA 3 or more for other reasons (chronic kidney disease, diabetes etc.)

\section{Exclusion criteria}

Irregular heart rhythm

Body weight less than $55 \mathrm{~kg}$ or more than $140 \mathrm{~kg}$

Age under 18 years

ASA, American Society of Anesthesiologists' physical status classification. 


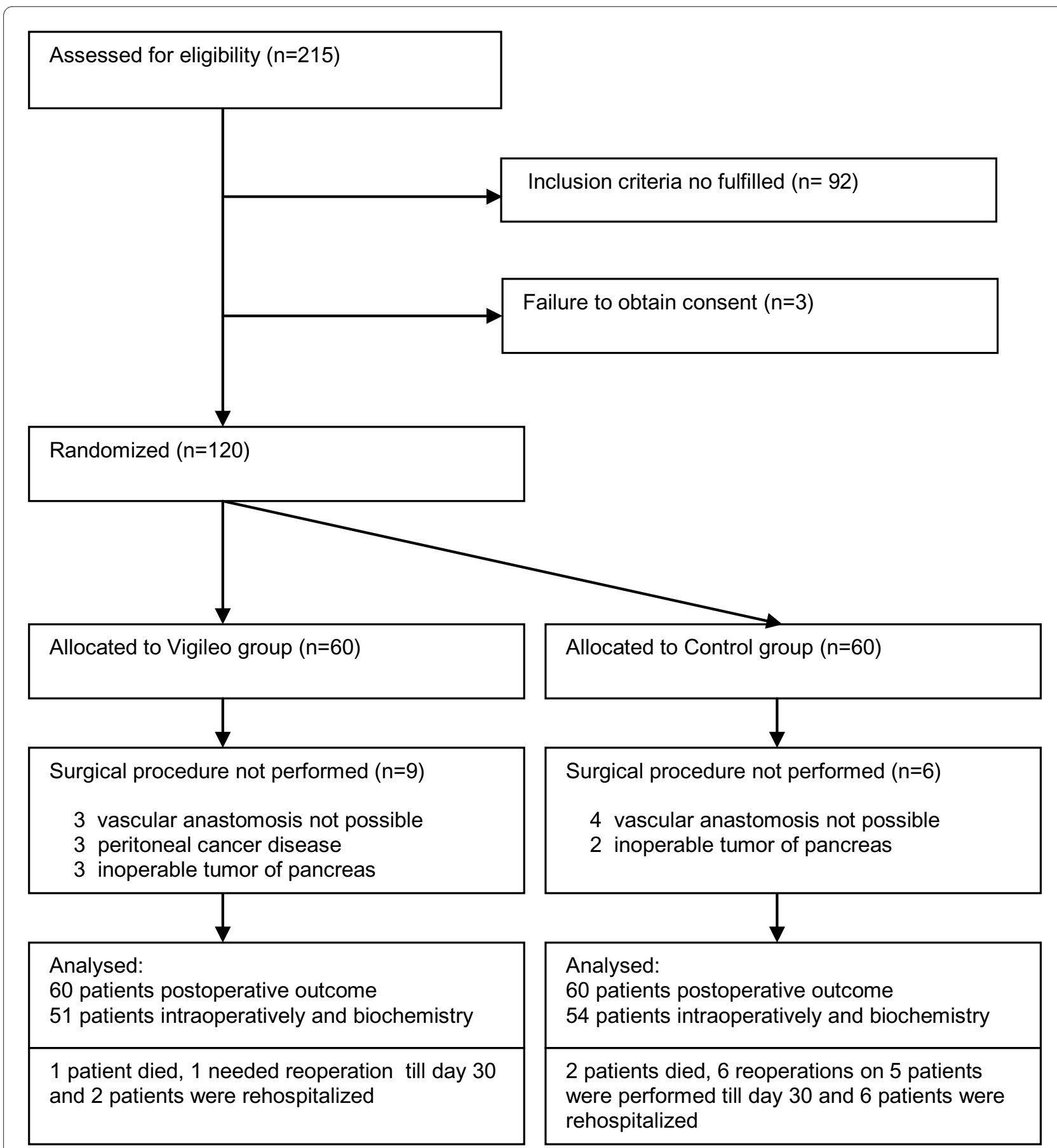

Figure 1 Flow of participants through the trial.

complications until day 30 after the operation, consistent with previous studies on peri-operative optimization $[11,12,16]$. Secondary outcome measures were hospital/ ICU length of stay and all-cause mortality. These parameters were assessed both on intention-to-treat and per protocol basis. Biochemical parameters of oxygen debt during operation and in early postoperative period (8 hours), that is serum lactate level, central venous oxygen saturation (ScvO2), arterial acid-base balance parameters and intraoperative hemodynamic parameters and amounts of intravenous fluids and inotropes used were assessed only in per protocol patients.

\section{Peri-operative care}

A central venous catheter was inserted via the subclavian or internal jugular vein the day before surgery. An antero- 
posterior chest $\mathrm{x}$-ray was obtained to exclude catheter malposition. Patients were premedicated according to institutional standards and an infusion of balanced crystalloid solution (Ringerfundin; B.Braun Melsungen Ag, Melsungen, Germany) at a rate of $2 \mathrm{ml} / \mathrm{kg} / \mathrm{hr}$ was started at $8 \mathrm{am}$ on the day of surgery. Baseline demographic parameters, blood pressure, and heart and respiratory rates as well as preoperative Acute Physiologic and Chronic Health Evaluation II (APACHE II) and Sequential Organ Failure Assessment (SOFA) scores were recorded in the operating room. Before induction of anesthesia, an arterial line (20G, BD Arterial Cannula, BD Critical Care Systems Ltd., Singapore) was inserted into the radial artery of the non-dominant forearm and first measurements and laboratory blood were taken. Optimal pressure signal damping was assessed using flush test before the first measurements. In the patients, who gave informed consent for epidural analgesia, a catheter for postoperative pain management was inserted between the Th7/8 and L1/2 vertebral interspaces and after performing a test for correct extradural placement, a dose of sufentanil 10 ug in $10 \mathrm{ml}$ saline solution was administered. Anesthesia was than induced using either thiopental $4 \mathrm{mg} / \mathrm{kg}$, propofol $2 \mathrm{mg} / \mathrm{kg}$ or etomidate $2 \mathrm{mg} / \mathrm{kg}$ in combination with sufentanil 5 to $15 \mathrm{ug}$. Tracheal intubation was facilitated by neuromuscular relaxation (atracurium, cis-atracurium or rocuronium), depending on comorbidities and anesthesiologists choice. Anesthesia was maintained with volatile anesthetics (sevoflurane or desflurane) in $\mathrm{N}_{2} \mathrm{O}$ and $\mathrm{O}_{2}$ mixture (0.9 to 1.2 MAC). Sufficient analgesia was provided using 5 ug boluses of sufentanil, or with a continuous infusion of sufentanil 10 ug and bupivacain $25 \mathrm{mg}$ in $20 \mathrm{ml}$ saline at a rate of 4 to 6 $\mathrm{ml} / \mathrm{hr}$ via an epidural catheter. All patients were mechanically ventilated with tidal volume $8 \mathrm{ml} / \mathrm{kg}$ and positive end-expiratory pressure (PEEP) $0.6 \mathrm{kPa}$, respiratory rate was set to maintain normocapnia. Anemia (hemoglobin level below $90 \mathrm{~g} / \mathrm{l}$ ) and acute blood loss higher than 20\% of calculated patient's circulatory volume were corrected with transfusions of packed red blood cells (RBC) and fresh frozen plasma (FFP), respectively. The number of transfused units (both RBC and FFP) was recorded as well as the amount of infused crystalloid and colloid solutions, diuresis and blood loss. At time of skin closure, blood was taken for acid-base balance analysis (both arterial and central venous), blood count and basic biochemical laboratory tests.

\section{Study protocol}

Vigileo/FloTrac device (Edwards Lifesciences, Irvine, CA, USA) with software version 1.10 was used for measuring SVV and other hemodynamic variables. Intraoperative basal fluid replacement was realized in both groups with continuous infusion of $8 \mathrm{ml} / \mathrm{kg} / \mathrm{hr}$ of crystalloid solution
(Plasmalyte; Baxter Czech spol.s.r.o, Praha, Czech Republic). In the Vigileo group, additional boluses of $3 \mathrm{ml} / \mathrm{kg}$ colloid solution (Voluven 130/0.4 6\%; Fresenius Kabi AG, Bad Homburg, Germany, Tetraspan 130/0.4 6\%; B.Braun Melsungen Ag, Melsungen, Germany) were given when SVV measured by Vigileo/FloTrac system rose above $10 \%$ (a sustained change during the previous five minutes) or in the case of positive response (cardiac index (CI) increase above $10 \%$ ) to previous fluid challenge. Central venous pressure (CVP) measurement served as a regulatory mechanism (Figure 2). An infusion of dobutamine was started to maintain CI between 2.5 and $4 \mathrm{l} / \mathrm{min} / \mathrm{m}^{2}$ under low cardiac output state conditions (CI less than $2.5 \mathrm{l} / \mathrm{min} / \mathrm{m}^{2}$ ) after appropriate fluid administration. Ephedrine boluses of 5 to $15 \mathrm{mg}$ or norepinephrine infusion were allowed in addition to colloid infusion to treat a fall in systolic arterial pressure below $90 \mathrm{mmHg}$ or mean arterial pressure (MAP) below $65 \mathrm{mmHg}$ (e.g. during clamp release or sudden large blood loss etc.) to maintain MAP above $70 \mathrm{mmHg}$. These episodes were recorded as hypotensive events and underwent analysis. In the Control group, the anesthesiologist was free to give additional fluids (both crystalloids and colloids) or use vasoactive substances to maintain blood pressure, diuresis and CVP in normal ranges (MAP $>65 \mathrm{mmHg}$, heart rate $<100$ bpm, CVP 8 to $15 \mathrm{mmHg}$, urine output $>0.5 \mathrm{ml} / \mathrm{kg} / \mathrm{h}$ ).

\section{Postoperative care and data collection}

After surgery, the patients were transferred to either ICU or a monitored bed on the standard ward. During the postoperative period the patients were managed by an intensivist or a surgeon, who was not aware of the patient's allocation in study group. Biochemical tests (arterial and central venous blood gas, serum lactate, blood count and other laboratory tests) were performed at 4,8 and 24 hours after the end of surgery. Basal measurements of blood pressure, heart and respiratory rates, peripheral hemoglobin oxygen saturation, diuresis, medication and intravenous fluids or blood products administered during eight hours postoperatively were retrospectively collected by a member of the research team blinded to patient allocation. Physiological and Operative Severity Score for the Enumeration of Mortality and Morbidity (POSSUM) [39] was calculated after operation along with SOFA score for the period of 8 and 24 hours postoperatively.

Patients were monitored up to discharge from the hospital for infectious and organ complications (cardiac, pulmonary, gastrointestinal, renal and thrombotic). The list of screened complications was based on the POSSUM scoring system and adapted according to other literature data $[11,12,16]$. Additionally, we followed complications deemed as life-threatening or disabling. Diagnosis and management of complications were undertaken by non- 
Inclusion of eligible patient and admission to OR

Randomization to Vigileo or Control group

Obtaining baseline physiologic variables

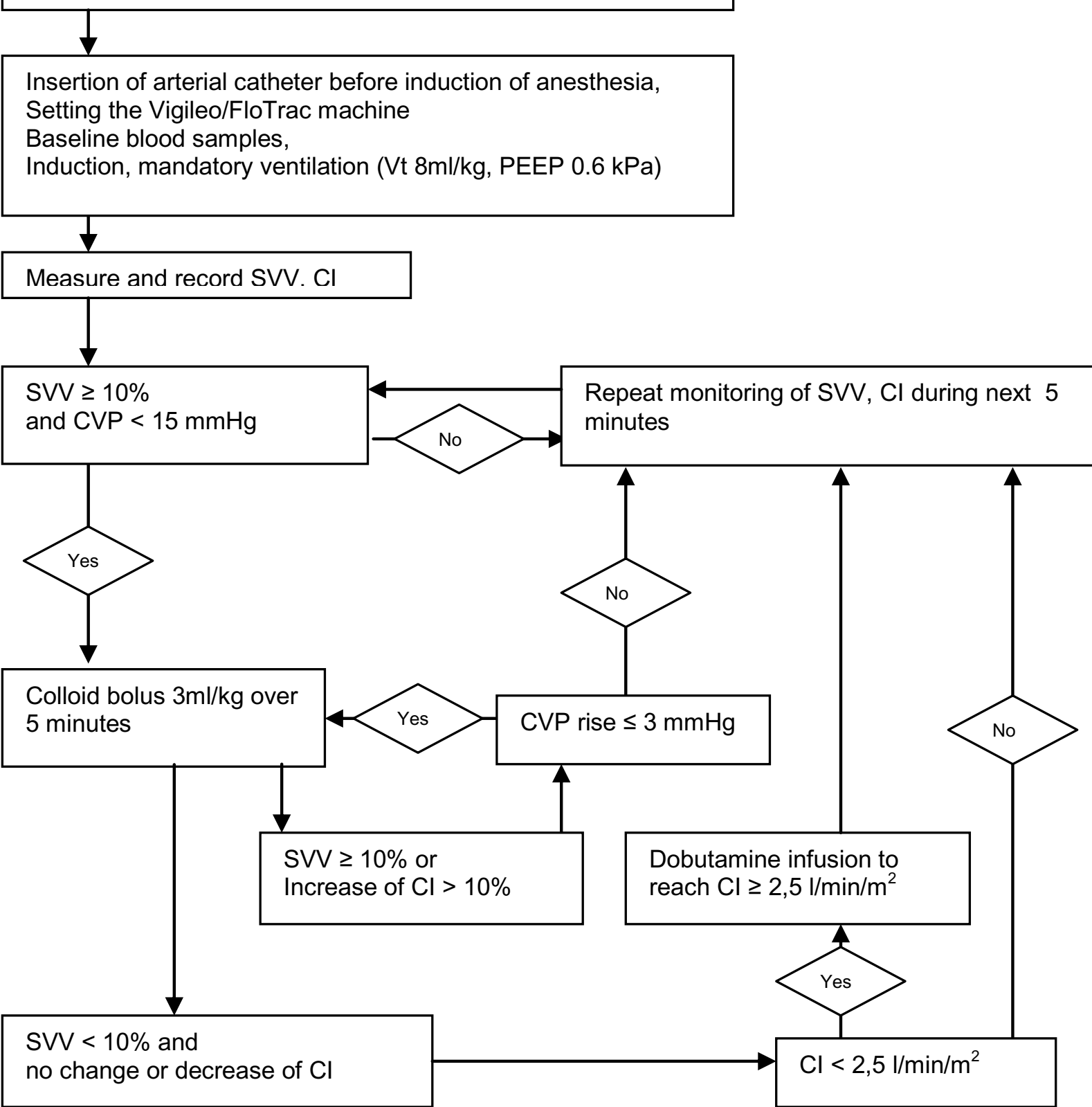

Figure 2 Fluid management in Vigileo group. Cl, cardiac index; CVP, central venous pressure; SW, stroke volume variation; PEEP, positive end-expiratory pressure; $\mathrm{Vt}$, tidal volume; $\mathrm{OR}$, operation room.

research staff. These were verified in accordance with predefined criteria $[40,41]$ where available by a member of the research team unaware of patient group allocation. Complications that occurred after discharge and required ambulatory or in-hospital care up to day 30 after surgery were also recorded. The total number of complications and the number of patients with complications were calculated for each group. The ICU and standard care length of stay and length of ventilator support were recorded at 
the end of hospitalization. If a patient died, the time from operation to death was recorded.

\section{Statistical analysis}

A high number of infectious and organ complications can be anticipated in high-risk surgical patients. According to our retrospective analysis of the incidence of complications in similar patient populations in our hospital $(65 \%$, unpublished data) and data from similar studies $[8,11,12]$, the percentage of patients with postoperative complications can exceed $60 \%$ with a 50 to $60 \%$ reduction described in intervention groups. For a decrease in morbidity from 65 to $38 \%$, a study sample size of 50 patients in each group was calculated for two-sided tests with type I error of $5 \%$ and power of $80 \%$. Owing to an anticipated loss of 15 to $20 \%$ of patients entering the study due to a change in scheduled surgery, we proposed to include 60 patients in each group. For a test of normal distribution, the Kolmogorov-Smirnov test was used. Continuous data with normal distribution were tested with paired or unpaired t-tests, non-normally distributed data using Mann-Whitney $U$ test and Wilcoxon rank-sum test for unpaired and paired results, respectively. The change in time-dependent variables was tested using analysis of variance (ANOVA) on repeated measurements or Friedman test. Categorical data were tested using chi-square test and chi-square test for trend. Unless stated otherwise, normally distributed data are presented as mean \pm (standard deviation), and as median (interquartile ranges) where not normally distributed. A $P<0.05$ was considered statistically significant for all tests. All calculations were performed with MedCalc version 10.4.8.0 (Frank Schoonjans, MedCalc Software, Broekstraat 52, 9030 Mariakerke, Belgium).

\section{Results}

A total of 215 patients were found to be eligible according to scheduled surgical procedures from July 2007 to May 2009. After examining these patients for inclusion criteria and obtaining informed consent, 120 patients were included and randomized to the Vigileo or Control groups. Fifteen patients dropped out after randomization because of unanticipated cancellation of their surgery (nine patients from the Vigileo group and six patients from the Control group). There were no other discontinuations or patients lost to follow-up (Figure 1).

Both groups were equal in basic demographic parameters, co-morbidities, American Society of Anesthesiologists' physical status classification status or surgical procedure performed. No significant differences in basal scoring systems (APACHE II, SOFA and POSSUM scores) at baseline were observed. Patients were also comparable in terms of baseline biochemical laboratory parameters and physiologic variables (Table 2).
The Vigileo group received a significant larger amount of colloid infusions (Vigileo $1,425 \mathrm{ml}(1,000$ to 1,500$)$ vs. Control 1,000 ml (540 to 1,250); $P=0.0028)$, the volume of infused crystalloids, the amount of blood products and blood loss did not differ between the groups. There was a trend towards maintaining higher diuresis during the study period in the Vigileo group (1.13 (0.76 to 1.85$) \mathrm{ml} /$ $\mathrm{kg} / \mathrm{hr}$ vs. 0.896 (0.56 to 1.43$) \mathrm{ml} / \mathrm{kg} / \mathrm{hr}$ in the Control group; $P=0.068)$. Lower incidence of intraoperative hypotensive events ( 2 ( 1 to 2 ) vs. 3.5 ( 2 to 6 ); $P=0.0001$ ) and a trend toward lower use of norepinephrine (3 patients $(5.88 \%)$ vs. 11 patients (20.37\%); $P=0.058$ ) was found in Vigileo group. The amount of fluids infused, diuresis, physiologic variables and pharmacological interventions within the first eight hours postoperatively did not significantly differ between the groups (Table 3). No difference in MAP, heart rate and CVP between the groups was observed at the end of surgery, although in both groups a significant decrease of MAP against baseline value was found. In the Vigileo group a decrease in heart rate ( $74 \pm 13$ vs. $70 \pm 11 ; P=0.0108)$, increase in CVP ( $8 \pm 2$ vs. $10 \pm 3 ; P=0.0002)$ from baseline occurred, while no such difference was observed in the Control group. At the end of surgery a decrease in SVV compared with preoperative value $(13 \pm 5$ vs. $7 \pm 2 ; P<0.0001)$ was observed in the Vigileo group, a similar parameter was not evaluated in the Control group.

An increase in serum lactate concentration was observed in the Control group compared with baseline at the end of surgery, four and eight hours after operation $(P$ $<0.01$, ANOVA on repeated measurements with Bonferroni correction). We found no such difference in the Vigileo group. Serum lactate concentration at the end of the operation in the Vigileo group was lower than in the Control group $(1.78 \mathrm{mmol} / \mathrm{l}$ vs. $2.25 \mathrm{mmol} / \mathrm{l} ; P=0.0252)$. Arterial $\mathrm{pH}$ decreased at the end of operation in both groups compared with baseline and normalized during postoperative period; however, in the Vigileo group the $\mathrm{pH}$ at the end of operation was higher (7.37 in the Vigileo vs. 7.35 in the Control groups; $P=0.049$ ). A concomitant decrease in base excess and serum bicarbonate from baseline at the end of surgery was observed in both groups but normalized early in the postoperative period, no difference was found between the groups. In comparison with baseline the $\mathrm{ScvO}_{2}$ in both groups was higher at the end of surgery and was lower 24 hours after operation (ANOVA on repeated measurement), no difference between the groups was observed. All results are presented in Table 4 and Figure 3.

Results of postoperative outcome are presented in Table 5 on an intention-to-treat basis and also as per protocol analysis. The incidence of organ and infectious complications in the 30-day postoperative period was lower in the Vigileo group (18 patients (30\%) vs. 35 
Table 2: Baseline demographics

\begin{tabular}{|c|c|c|c|}
\hline Parameters & Vigileo group & Control group & $P$ value \\
\hline Number in group & 60 & 60 & \\
\hline Male : Female & $50: 10$ & $47: 13$ & 0.643 \\
\hline Age & $66.73 \pm 7.88$ & $66.32 \pm 8.38$ & 0.78 \\
\hline Weight (kg) & $80.47 \pm 12.75$ & $82.49 \pm 17.18$ & 0.466 \\
\hline Height (cm) & $172.07 \pm 7.2$ & $172.1 \pm 10.19$ & 0.99 \\
\hline APACHE II score & $6.59 \pm 3.04$ & $6.76 \pm 2.61$ & 0.758 \\
\hline SOFA score & $1(1-2)$ & $1(0-2)$ & 0.82 \\
\hline POSSUM (operative score) & $17(16-20)$ & $17(14-20)$ & 0.304 \\
\hline POSSUM (physiology score) & $20(19-23)$ & $21(19-23)$ & 0.295 \\
\hline ASA (1:2:3:4:5) & 0:14:37:9:0 & 0:11:40:9:0 & 0.646 \\
\hline \multicolumn{4}{|l|}{ Chronic disease } \\
\hline Coronary artery disease & $32(53 \%)$ & $31(52 \%)$ & 0.942 \\
\hline Hypertension & $56(93 \%)$ & $56(93 \%)$ & 0.721 \\
\hline Peripheral artery disease & $31(52 \%)$ & $30(50 \%)$ & 0.971 \\
\hline COPD/Asthma bronchiale & $13(22 \%)$ & $12(20 \%)$ & 0.964 \\
\hline $\begin{array}{l}\text { Other pulmonary } \\
\text { pathology }\end{array}$ & $5(8 \%)$ & $3(5 \%)$ & 0.767 \\
\hline Cerebrovascular disease & $8(13 \%)$ & $8(13 \%)$ & 0.786 \\
\hline Diabetes mellitus & $21(35 \%)$ & $23(38 \%)$ & 0.851 \\
\hline Chronic kidney disease & $5(8 \%)$ & $4(7 \%)$ & 0.89 \\
\hline Malignancy & $23(38 \%)$ & $23(38 \%)$ & 0.851 \\
\hline Age $>70$ years & $24(40 \%)$ & $21(35 \%)$ & 0.706 \\
\hline \multicolumn{4}{|l|}{ Surgical procedure } \\
\hline Colo-rectal surgery & $17(28 \%)$ & $16(27 \%)$ & 0.935 \\
\hline Pancreatic surgery & $5(8 \%)$ & $3(5 \%)$ & 0.767 \\
\hline $\begin{array}{l}\text { Intraabdominal vascular } \\
\text { surgery }\end{array}$ & $38(63 \%)$ & $41(68 \%)$ & 0.701 \\
\hline Surgery cancelled (Figure 1) & $9(15 \%)$ & $6(10 \%)$ & 0.581 \\
\hline Epidural analgesia & $35(58 \%)$ & $37(62 \%)$ & 0.794 \\
\hline
\end{tabular}

Values are presented as absolute (percentage), mean \pm standard deviation or median (interquartile range).

APACHE II, Acute Physiology And Chronic Health Evaluation score Il; ASA, American Society of Anesthesiologists' physical status classification; COPD, chronic obstructive pulmonary disease; POSSUM, physiologic and operative severity score for the enumeration of mortality and morbidity score; SOFA, Sequential Organ Failure Assessment score.

(58.3\%); $\mathrm{P}=0.0033$; relative risk $=0.514 ; 95 \%$ confidence interval $=0.331$ to 0.8 ) and also the number of complications was significantly diminished ( 34 vs. 77; $\mathrm{P}=0.0066$ ). The incidence of severe complications (7 patients $(11.7 \%)$ vs. $22(36.7 \%)$; $\mathrm{P}=0.0028$; relative risk $=0.318$; $95 \%$ confidence interval $=0.147$ to 0.688$)$ and their number $(13$ complications vs. $41 ; \mathrm{P}=0.0132$ ) was also lower in the Vigileo group. There was no difference in mortality, hospital and ICU length of stay between groups. Similar results were obtained when analyzing only patients whose optimization was carried out: complication rate was lower in Vigileo (16 patients (31.37\%) vs. 32 (59.26\%);
$\mathrm{P}=0.0076$; relative risk $=0.5294 ; 95 \%$ confidence interval $=0.3335$ to 0.8405$)$ as well as their number ( 32 vs. $73 ; \mathrm{P}=$ 0.0141). Severe complications developed six patients $(11.76 \%)$ in Vigileo vs. 19 (35.19\%) in the Control group $(\mathrm{P}=0.0097$; relative risk $=0.3344 ; 95 \%$ confidence inter$\mathrm{val}=0.1452$ to 0.7701$)$ and their number (12 complications vs. $38 ; \mathrm{P}=0.0274$ ) was also lower in the Vigileo group. In this per protocol group a lower rate of complications was even associated with shorter hospital length of stay in the Vigileo group (9 (8 to 12) vs. 10 (8 to 19); $\mathrm{P}=$ 0.0421 ). Again there was no difference in mortality and ICU length of stay. 
Table 3: Perioperative period ('per protocol' analysis)

\begin{tabular}{|c|c|c|c|}
\hline \multirow[t]{2}{*}{ Parameters } & \multirow{2}{*}{$\begin{array}{l}\text { Vigileo group } \\
n=51(85 \%)\end{array}$} & \multirow{2}{*}{$\begin{array}{l}\text { Control group } \\
n=54(90 \%)\end{array}$} & \multirow[t]{2}{*}{$P$ value } \\
\hline & & & \\
\hline \multicolumn{4}{|l|}{ Baseline measurement } \\
\hline $\mathrm{MAP}(\mathrm{mmHg})$ & $103 \pm 13$ & $103 \pm 16$ & 0.948 \\
\hline CVP $(\mathrm{mmHg})$ & $8 \pm 2$ & $9 \pm 4$ & 0.362 \\
\hline HR (beats/min) & $74 \pm 13$ & $74 \pm 10$ & 0.851 \\
\hline $\mathrm{Cl}\left(\mathrm{ml} / \mathrm{min} / \mathrm{m}^{2}\right)$ & $3 \pm 0.64$ & N/A & \\
\hline SVV (\%) & $13 \pm 5$ & $\mathrm{~N} / \mathrm{A}$ & \\
\hline \multicolumn{4}{|l|}{ End of surgery } \\
\hline $\mathrm{MAP}(\mathrm{mmHg})$ & $92 \pm 12^{*}$ & $91 \pm 14^{*}$ & 0.702 \\
\hline \multirow[t]{2}{*}{$\mathrm{CVP}(\mathrm{mmHg})$} & $10 \pm 3^{*}$ & $10 \pm 3$ & 0.439 \\
\hline & ${ }^{*}(P=0.0002$ vs. baseline $)$ & & \\
\hline \multirow[t]{2}{*}{ HR (beats/min) } & $70 \pm 11^{*}$ & $73 \pm 15$ & 0.264 \\
\hline & $*(P=0.0108$ vs. baseline $)$ & & \\
\hline \multirow[t]{2}{*}{$\mathrm{Cl}\left(\mathrm{ml} / \mathrm{min} / \mathrm{m}^{2}\right)$} & $3.6 \pm 0.7^{*}$ & $\mathrm{~N} / \mathrm{A}$ & \\
\hline & ${ }^{*}(P<0.0001$ vs. baseline $)$ & & \\
\hline \multirow[t]{2}{*}{ SVV (\%) } & $7 \pm 2^{*}$ & N/A & \\
\hline & ${ }^{*}(P<0.0001$ vs. baseline $)$ & & \\
\hline Number of hypotensive periods intraoperatively & $2(1-2)$ & $3.5(2-6)$ & 0.0001 \\
\hline Length of anesthesia (min) & $184 \pm 46$ & $202 \pm 53$ & 0.072 \\
\hline Length of surgery (min) & $163 \pm 44$ & $176 \pm 55$ & 0.214 \\
\hline Length of aortic cross-clamping & $61.5 \pm 35$ & $57 \pm 35$ & 0.592 \\
\hline Crystalloids (ml) & $2321 \pm 681$ & $2459 \pm 930$ & 0.386 \\
\hline Colloids (ml) & $1425(1000-1500)$ & $1000(540-1250)$ & 0.0028 \\
\hline Blood (ml) & $0(0-566)$ & $270(0-578)$ & 0.633 \\
\hline Fresh frozen plasma (ml) & $0(0-540)$ & $0(0-540)$ & 0.793 \\
\hline Estimated blood loss (ml) & $700(500-1200)$ & $800(400-1325)$ & 0.511 \\
\hline Diuresis $(\mathrm{ml} / \mathrm{kg} / \mathrm{h})$ & $1.13(0.76-1.85)$ & $0.896(0.56-1.43)$ & 0.068 \\
\hline Norepinephrine & $3(5.88 \%)$ & $11(20.37 \%)$ & 0.058 \\
\hline Dobutamine & $2(3.92 \%)$ & $0(0 \%)$ & 0.451 \\
\hline Vasodilatation therapy & $5(9.8 \%)$ & $3(5.56 \%)$ & 0.652 \\
\hline \multicolumn{4}{|l|}{ After eight hours on ICU } \\
\hline Crystalloids (ml) & $1587 \pm 371$ & $1528 \pm 475$ & 0.485 \\
\hline Colloids (ml) & $0(0-500)$ & $0(0-250)$ & 0.887 \\
\hline Blood (ml) & $0(0-0)$ & $0(0-0)$ & 0.746 \\
\hline Fresh frozen plasma (ml) & $0(0-0)$ & $0(0-0)$ & 0.744 \\
\hline Diuresis $(\mathrm{ml} / \mathrm{kg} / \mathrm{h})$ & $1.18(0.79-1.89)$ & $1.08(0.83-1.89)$ & 0.886 \\
\hline Norepinephrine & $7(13.72 \%)$ & $6(11.11 \%)$ & 0.913 \\
\hline Dobutamine & $1(1.96 \%)$ & $0(0 \%)$ & 0.977 \\
\hline Vasodilatation therapy & $10(19.61 \%)$ & $9(16.67 \%)$ & 0.891 \\
\hline Diuretic support & $13(25.49 \%)$ & $17(31.48 \%)$ & 0.643 \\
\hline SOFA (8 hours) & $3(2-5.25)$ & $3(1-4)$ & 0.474 \\
\hline SOFA (24 hours) & $2(2-4)$ & $3(1.5-4)$ & 0.541 \\
\hline
\end{tabular}

Perioperative period analyzed only for patients whose intraoperative protocol was carried in full extent. Values are presented as absolute (percentage), mean \pm standard deviation or median (interquartile range).

$\mathrm{Cl}$, cardiac index; CVP, central venous pressure; HR, heart rate; MAP, mean arterial pressure; N/A, not applicable; SOFA, Sequential Organ Failure Assessment; SVV, stroke volume variation. 
When analyzing patients according to developed complications, a higher serum lactate level at the end of surgery (1.5 (1.2 to 2.2$)$ vs. $2 \mathrm{mmol} / \mathrm{l}(1.4$ to 2.8$) ; P=0.0084)$ and four hours after (1.4 (1 to 2 ) vs. $2 \mathrm{mmol} / \mathrm{l}$ (1.4 to 3.1 ); $P=0.003)$ was observed in the group with complications. Severe complications were associated with lower $\mathrm{CI}$ at the end of surgery ( $3.7 \pm 0.7$ vs. $2.95 \pm 0.3 ; P=0.0108)$. No difference in postoperative $\mathrm{ScvO} 2$ or other laboratory and hemodynamic parameters was found. Length of stay in ICU (3 (0 to 4 ) vs. four days ( 2 to 6$) ; P=0.0054$ ) and in hospital (8 (7 to 10$)$ vs. 11 days ( 8 to 16.8$) ; P<0.0001$ ) was shorter in the group without complications.

\section{Discussion}

Intraoperative fluid optimization in high-risk surgical patients undergoing major abdominal surgery using SVV and Vigileo/FloTrac monitor increased hemodynamic stability during operation, decreased lactate concentration at the end of operation and was associated with a lower rate of postoperative complications with a tendency to decrease hospital length of stay.

To our knowledge, this is the first study using a Vigileo/ FloTrac monitor in the perioperative setting for guiding fluid management mainly by SVV. Recently a study by
Mayer and colleagues [42], looking at a group of surgical patients, was optimized using Vigileo/FloTrac was published with very similar results regarding postoperative complication rates and counts. Also, the same software version (second generation - 1.10) was used. Including Vigileo software version information is of critical importance, as second-generation devices seem to be more accurate according to some studies [31-35] although many controversial results have been presented recently. Reliability of the device was questioned in hemodynamically unstable patients [43], in those with high heart rate variability or when sudden changes in vascular tone occur as in cases of vasoactive drugs bolus administration etc [36,37], or during hepatic surgery [44,45]. Specifically, the influence of systemic vascular resistance alteration on accuracy of the Vigileo monitor is of note and might be a source of possible bias, particularly in patients under general anesthesia. Systemic vascular resistance was measured in the Vigileo group and no significant divergences from normal ranges were observed. Furthermore, the use and dosage of vasopressors was relatively low in our patients. Moreover, a sustained change of hemodynamic parameters was one of the conditions in the decision protocol to minimize these flaws. The aim of our study was

Table 4: Biochemical variables

\begin{tabular}{|c|c|c|c|c|c|c|}
\hline Variable & Baseline & End of surgery & $\begin{array}{l}4 \text { hours } \\
\text { postOP }\end{array}$ & $\begin{array}{l}8 \text { hours } \\
\text { postOP }\end{array}$ & $\begin{array}{l}24 \text { hours } \\
\text { postOP }\end{array}$ & $\begin{array}{l}\text { ANOVA on } \\
\text { rpt.m. }\end{array}$ \\
\hline \multicolumn{7}{|c|}{ Serum lactate ( $\mathrm{mmol} / \mathrm{l})$} \\
\hline Vigileo & $1.48 \pm 0.44$ & $1.78 \pm 0.83^{\#}$ & $1.75 \pm 0.86$ & $1.85 \pm 0.98$ & $1.25 \pm 0.52$ & 0.002 \\
\hline Control & $1.42 \pm 0.43$ & $2.25 \pm 1.12 * * *$ & $2.14 \pm 1.11^{* * *}$ & $2.10 \pm 1.18^{* *}$ & $1.4 \pm 0.50$ & $<0.001$ \\
\hline \multicolumn{7}{|c|}{ Arterial pH } \\
\hline Vigileo & $7.43 \pm 0.03$ & $7.37 \pm 0.05 * * * \#$ & $7.39 \pm 0.04^{* *}$ & $7.41 \pm 0.05$ & $7.41 \pm 0.03^{*}$ & $<0.001$ \\
\hline Control & $7.41 \pm 0.04$ & $7.35 \pm 0.05 * * *$ & $7.38 \pm 0.05^{* *}$ & $7.40 \pm 0.05$ & $7.42 \pm 0.04$ & $<0.001$ \\
\hline \multicolumn{7}{|c|}{ Base excess (mmol/l) } \\
\hline Vigileo & $0.67 \pm 1.72$ & $-1.55 \pm 1.91^{* * *}$ & $-0.23 \pm 2.19^{*}$ & $0.41 \pm 1.8$ & $1.36 \pm 2.36$ & $<0.001$ \\
\hline Control & $-0.19 \pm 2.55$ & $-2.15 \pm 2.54^{* * *}$ & $-0.55 \pm 2.44$ & $-0.09 \pm 2.64$ & $1.17 \pm 2.17$ & $<0.001$ \\
\hline \multicolumn{7}{|c|}{ Serum bicarbonate (mmol/l) } \\
\hline Vigileo & $24.63 \pm 1.81$ & $23.05 \pm 1.68^{* * *}$ & $24.11 \pm 2.36$ & $24.65 \pm 1.84$ & $25.59 \pm 2.59$ & $<0.001$ \\
\hline Control & $23.81 \pm 2.69$ & $22.67 \pm 2.16^{* *}$ & $24.04 \pm 2.28$ & $24.33 \pm 2.57$ & $25.45 \pm 2.94$ & $<0.001$ \\
\hline \multicolumn{7}{|c|}{ ScvO2 (\%) } \\
\hline Vigileo & $71.79 \pm 6.94$ & $80.18 \pm 7.86^{* * *}$ & $69.43 \pm 8.40$ & $68.54 \pm 8.23$ & $67.61 \pm 6.54^{*}$ & $<0.001$ \\
\hline Control & $72.27 \pm 6.77$ & $80.04 \pm 6.87^{*}$ & $69.00 \pm 7.92$ & $69.50 \pm 7.84$ & $67.36 \pm 7.14^{* *}$ & $<0.001$ \\
\hline \multicolumn{7}{|c|}{ Hemoglobin (g/dl) } \\
\hline Vigileo & $12.3 \pm 1.5$ & $10.5 \pm 1.1^{* * *}$ & $11.4 \pm 1.5^{* * * *}$ & $11.2 \pm 1.5^{* * *}$ & $10.7 \pm 1.3^{* * *}$ & $<0.001$ \\
\hline Control & $12.7 \pm 1.6$ & $10.4 \pm 1.2^{* * *}$ & $11.4 \pm 1.3^{* * *}$ & $11.2 \pm 1.4^{* * *}$ & $10.8 \pm 1.0^{* * *}$ & $<0.001$ \\
\hline
\end{tabular}

Perioperative period analyzed only for patients whose intraoperative protocol was carried in full extent; ${ }^{*} P<0.05{ }^{* * P} P<0.01{ }^{* * * P} P<0.001$ analysis of variance (ANOVA) on repeated measurements with Bonferroni correction against baseline; $\# P<0.05$ Vigileo vs. Control ( $\mathrm{t}$-test); Values are presented as mean \pm standard deviation.

PostOP, postoperatively; ScvO2, central venous oxygen saturation. 


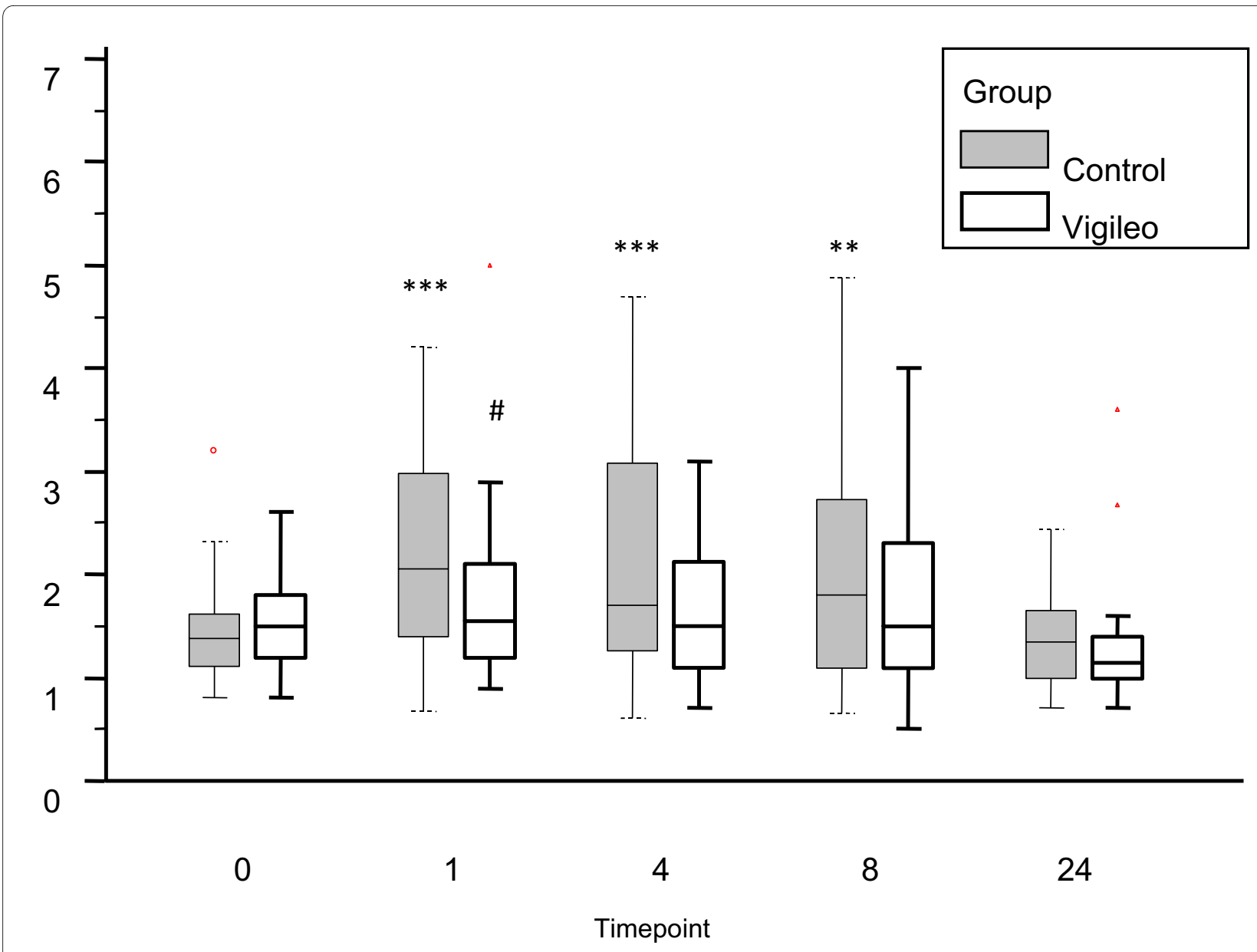

Figure 3 Serum lactate concentrations ( $\mathbf{m m o l} / \mathbf{l}){ }^{* *} P<0.01,{ }^{* *} P<0.001$ analysis of variance on repeated measurements against baseline; $\# P<$ 0.05 Vigileo vs. Control (t-test).

to evaluate the clinical utility of this safe and easy-to-use device. Using a new software generation (version 3.0 or higher) would probably enhance the monitor performance, but it was not available at the beginning of our study.

Using dynamic variables including SVV, some possible confounders should be considered. We already mentioned the influence of tidal volume [24,25], heart rhythm [38] and use of vasopressors [26]. We tried to minimize all of these as described by using a fixed tidal volume of 8 $\mathrm{ml} / \mathrm{kg}$ with PEEP $0.6 \mathrm{kPa}$ and excluding patients with irregular heart rhythm. As mentioned,a sustained rise of SVV above $10 \%$ in a period of five minutes was needed to start an intervention in order to exclude a possible bias due to surgical manipulations or other influences. We used the $10 \%$ threshold proposed by Manecke [27], which was the best available recommended value for Vigileo/ FloTrac at the time of preparing our protocol, although the optimal cut-off value for SVV is still uncertain. A study in cardiosurgical patients [21] proposed a lower target of 9.6\%, although other trials in patients undergoing major abdominal surgery [22] offered a more liberal value of $12 \%$. Another study [36] was unable to find a good predictive cut-off value under open abdomen conditions, probably showing that some hidden confounder still exists. These inconclusive results show that a further evaluation of dynamic variables is needed and results of protocols based only on variations itself should be assessed with caution. We used a dynamic change of CI and CVP for decision-making as safety measures to forestall these potential flaws.

Two studies were published using dynamic variables for intraoperative fluid management. Lopes and colleagues [11] demonstrated a significant morbidity reduction using solely pulse pressure variation in the optimization of high-risk surgical patients with results similar to our study and other literature concerning GDT [18,27]. A major limitation of that study was the small number of patients included (17 patients optimized and 16 in the control group). The complications rate was high ( $75 \%$ vs. 
Table 5: Summary of outcomes and complications

\begin{tabular}{|c|c|c|c|}
\hline Parameters & Vigileo group & Control group & $P$ value \\
\hline \multicolumn{4}{|l|}{ Number of patients } \\
\hline ITT analysis & 60 & 60 & \\
\hline Per protocol analysis & 51 & 54 & \\
\hline \multicolumn{4}{|l|}{ Mortality (\%) } \\
\hline $\mathrm{ITT}$ & $1(1.67 \%)$ & $2(3.33 \%)$ & 1.0 \\
\hline Per protocol & $1(1.96 \%)$ & $1(1.85 \%)$ & 0.501 \\
\hline \multicolumn{4}{|l|}{ Hospital length of stay (days) } \\
\hline $\mathrm{ITT}$ & $9(8-11.5)$ & $10(8-16)$ & 0.0937 \\
\hline Per protocol & $9(8-12)$ & $10(8-19)$ & 0.0421 \\
\hline \multicolumn{4}{|l|}{ ICU length of stay (days) } \\
\hline $\mathrm{ITT}$ & $3(2-5)$ & $3(0.5-5)$ & 0.789 \\
\hline Per protocol & $3(2-6)$ & $3(2-5)$ & 0.368 \\
\hline Rehospitalization (ITT only) & $2(3.33 \%)$ & $6(10 \%)$ & 0.272 \\
\hline \multicolumn{4}{|l|}{ Morbidity (day 30) } \\
\hline \multicolumn{4}{|l|}{ Patients with complications } \\
\hline $\mathrm{ITT}$ & $18(30 \%)$ & 35 (58.3\%) & 0.0033 \\
\hline Per protocol & $16(31.37 \%)$ & $32(59.26 \%)$ & 0.0076 \\
\hline \multicolumn{4}{|l|}{ Patient with severe complication $\left({ }^{*}\right)$} \\
\hline $\mathrm{ITT}$ & $7(11.7 \%)$ & $22(36.6 \%)$ & 0.0028 \\
\hline Per protocol & $6(11.76 \%)$ & 19 (35.19\%) & 0.0097 \\
\hline \multicolumn{4}{|l|}{ Complications (day 30) } \\
\hline $\mathrm{ITT}$ & 34 & 77 & 0.0066 \\
\hline Per protocol & 32 & 73 & 0.0141 \\
\hline \multicolumn{4}{|l|}{ Severe complications (day 30$)\left({ }^{*}\right)$} \\
\hline $\mathrm{ITT}$ & 13 & 41 & 0.0132 \\
\hline Per protocol & 12 & 38 & 0.0274 \\
\hline \multicolumn{4}{|l|}{ List of complications (ITT only) } \\
\hline \multicolumn{4}{|l|}{ Infectious } \\
\hline Pneumonia * & 4 & 8 & \\
\hline Sepsis * & 1 & 8 & \\
\hline Intraabdominal infection * & 1 & 4 & \\
\hline Catheter-related bloodstream inf. ${ }^{*}$ & 1 & 8 & \\
\hline Urinary tract infection & 3 & 13 & \\
\hline Wound infection/dehiscence & 2 & 5 & \\
\hline Decubital inf. & 1 & 3 & \\
\hline \multicolumn{4}{|l|}{ Cardiovascular } \\
\hline Arrhythmias (non-life threatening) & 3 & 5 & \\
\hline Arrhythmias (life threatening) ${ }^{*}$ & 0 & 0 & \\
\hline Heart failure/Pulmonary edema * & 3 & 6 & \\
\hline Acute myocardial infarction * & 0 & 1 & \\
\hline \multicolumn{4}{|l|}{ Respiratory } \\
\hline Pulmonary embolism * & 0 & 0 & \\
\hline
\end{tabular}


Table 5: Summary of outcomes and complications (Continued)

\begin{tabular}{|c|c|c|}
\hline ALI/ARDS * & 0 & 0 \\
\hline Ventilator support (incl.postoperative) & 3 & 6 \\
\hline New onset of ventilator support * & 2 & 4 \\
\hline \multicolumn{3}{|l|}{ Renal } \\
\hline AKI (without dialysis) & 2 & 4 \\
\hline Renal failure with dialysis * & 1 & 1 \\
\hline \multicolumn{3}{|l|}{ Thrombotic } \\
\hline Stroke (including TIA) * & 0 & 1 \\
\hline Deep vein thrombosis & 0 & 1 \\
\hline Graft thrombosis & 1 & 3 \\
\hline \multicolumn{3}{|l|}{ Gastro-intestinal } \\
\hline GIT bleeding & 0 & 0 \\
\hline GIT obstruction & 0 & 0 \\
\hline Pancreatitis (edematous/necrotizing *) & $2 / 0$ & $0 / 0$ \\
\hline Hepatic dysfunction (mild/severe *) & $0 / 0$ & $1 / 0$ \\
\hline
\end{tabular}

41\%) compared with our results but the proportional reduction was similar. One possible explanation for this disproportion is a higher number of ASA 4 patients. A second study [46] found no treatment benefit using systolic pressure variation (SPV) in guiding fluid management. The reason for different results could lie in the population studied being much healthier and a relatively liberal use of norepinephrine. As SPV is more influenced by afterload modification than SVV [47], patients in this study could have been, despite vigorous fluid resuscitation, still not optimized. This opinion is supported by a high SVV in the study group (12\% in 3rdhour and $11 \%$ in 6thhour of protocol) compared with low SPV (7\% and 6\% in the same time points).

The rate and number of postoperative complications in our study were significantly lower in the interventional group. This reduction corresponds with many GDT trials including the recently published study by Mayer and colleagues [42], where only $20 \%$ of GDT patients developed complications compared with $50 \%$ in the control group. GDT is generally associated with infusion of larger amounts of colloids and improvement in hemodynamic parameters at the end of surgery. A difference between GDT and control groups are described in some studies, but neither the current trial or the one by Mayer and colleagues brings information about more detailed hemodynamic parameters in control groups whose management was guided with standard care (CVP, MAP etc). This naturally limits interpretation about whether the SVVguided fluid loading is really the cause of morbidity reduction. Nevertheless, the one of the most probable causes of GDT success is a timely recognition of hemodynamic derangements and prompt intervention for their solution. Such an effect may not lead to a significant difference of hemodynamic variables at the end of surgery, but more probably would manifest in biochemical markers resulting from these derangements.

Biochemical parameters of oxygen debt (serum lactate level, its normalization and low ScvO2 or low mixed venous oxygen saturation ( $\mathrm{SvO} 2)$ ) could serve as these markers and are early indicators of unfavorable outcome in critically ill patients $[4,48,49]$. In our study we observed an increase in serum lactate concentration in the Control group, which was associated with arterial $\mathrm{pH}$ decrease and persisted for eight hours postoperatively. The course of $\mathrm{ScvO} 2$ values at different time points was similar in both groups with a slight elevation during anesthesia and decrease 24 hours after operation. A difference in arterial serum lactate level between patients with complications and those without was detected. A higher mean lactate 24 hours postoperatively in the study by Lopes and colleagues [11] and Chytra and colleagues [9] showed that GDT decreased lactate level with a possible association to a reduction of infectious complications. A good predictive value of postoperative $\mathrm{ScvO} 2$ was found by Pearse and colleagues [48] in the analysis of their GDT study with a proposed cut-off value of $64.4 \%$, and another study [49] found even higher predictive ScvO2 levels of 73\%. We were unable to prove any correlation between ScvO2 and postoperative morbidity. A large portion of vascular 
surgery procedures (above $60 \%$ in both groups) probably contributed to this phenomenon. Lactate generated during aortic cross clamping in lower body parts spread after clamp release into systemic circulation. Hemodynamic instability and low intravascular volume during the reperfusion period could delay restitution of normal flow promoting ischemia-reperfusion injury with consequent complications. This may impact on the fidelity of $\mathrm{ScvO}_{2}$ and arterial serum lactate levels might be a better predictor of outcome under these circumstances. Lactate-free fluids were used for volume substitution to exclude potential bias and the time of ischemia producing aortic cross-clamping was measured and was comparable between groups.

In contrast to the lower incidence and number of complications a limited impact on the length of stay in the ICU or hospital was found in our study. Only hospital length of stay in those patients whose optimization protocol was carried out was reduced. However, some factors can limit the generalization of these findings. Each institution usually has its own regimens and protocols of ICU and ward care, which can significantly change the length of stay. A 'medical fitness for discharge' was used by some authors $[8,13,16]$ to overcome the problem of social hospitalization and other biases. Discharge criteria were not predefined in our study, which can limit the interpretation of both (hospital and ICU) length of stay parameters. Other possible reasons why the ICU length of stay was similar in both groups, although rate of severe complications was higher in the controls, is a suspected 'overtreatment' of patients without complications (a median of three days on ICU was observed in uncomplicated patients). When comparing other GDT studies the hospital length of stay is similar to our results in both groups $[7,8,11,14]$. Some other factors such as mobilization of patients or suture removal after laparotomy can limit and influence length of stay on the surgical ward more than medical fitness to discharge. A higher proportion of rehospitalized patients in the control group (3.3\% vs. $10 \%$; although not statistically significant) could support this notion. Low mortality counts were observed in both groups in comparison with other authors [11,12,42], but similar to those proposed by POSSUM (Portsmouth modification) and APACHE II scoring systems (both $2.82 \%)$. Inclusion of emergency patients [12] and a higher proportion of ASA grade 4 patients [11] or possibly an older population and more complex surgical procedures [42] in other studies can also explain this difference.

The single-center design belongs to major limitations of the trial. The potential bias resulting from institutional standards of care has already been discussed. Also the inclusion of a mixture of surgical procedures could influence our results, because the pathophysiology and causes of complications vary between vascular and non-vascular major abdominal surgery. Our goal was to evaluate the optimization protocol on a nonspecific surgical population usually treated in our institution. We conducted a retrospective analysis of patients undergoing similar surgical procedures and proposed a suitable group size in order to reach the predefined goal of morbidity reduction. A better understanding of observed relations would be possible with a more homogenous population or by a subgroup analysis of a larger sample. Also our study lacks power to show a significant reduction in mortality. On the contrary the extensive reduction in morbidity in such a small population advocates the value of this relatively simple intervention; however, it will have to be proven in a larger multi-center study.

\section{Conclusions}

Optimization of intravascular volume during major abdominal surgery using SVV and Vigileo cardiac output monitor is associated with better intraoperative hemodynamic stability and decrease in serum lactate concentration at the end of surgery. In the postoperative period a significantly lower incidence of complications were found. A larger and multicenter study, optimally using the novel software generation should be performed to confirm results of our study.

\section{Key messages}

- In this study, intraoperative hemodynamic optimization using SVV in high-risk patients undergoing major abdominal surgery was associated with improved hemodynamic stability and reduced serum lactate concentration at the end of surgery.

- In this study, GDT using SVV as an end-point was associated with reduced postoperative complication rates.

\section{Abbreviations}

APACHE: Acute Physiologic and Chronic Health Evaluation; ANOVA: analysis of variance; ASA: American Society of Anesthesiologists'physical status classification; Cl: cardiac index; CVP: central venous pressure; FFP: fresh frozen plasma; GDT: goal-directed therapy; MAP: mean arterial pressure; PEEP: positive endexpiratory pressure; POSSUM: Physiological and Operative Severity Score for the Enumeration of Mortality and Morbidity; RBC: red blood cells; ScvO2: central venous oxygen saturation; SOFA: Sequential Organ Failure Assessment; SPV: systolic pressure variation; SW: stroke volume variation.

\section{Competing interests}

The authors declare that they have no competing interests.

\section{Authors' contributions}

$\mathrm{JB}, \mathrm{ICH}, \mathrm{EK}$ and RP were responsible for the study design. JB, PA, MH, RS and MS were responsible for administering the protocol. JB and ICH provided the data analysis and drafting the manuscript. All authors have given final approval of this version of the manuscript.

\section{Acknowledgements}

The authors would like to thank the nursing staff at University Hospital PIzen for their assistance with this study. The study was supported by a research grant of Czech Ministry of Education MSM0021620819 


\section{Author Details}

Department of Anesthesiology and Intensive Care, Charles University teaching hospital, alej Svobody 80, PIzen, 304 60, Czech Republic

Received: 12 January 2010 Revised: 4 May 2010

Accepted: 16 June 2010 Published: 16 June 2010

\section{References}

1. Bundgaard-Nielsen $\mathrm{M}$, Holte $\mathrm{K}$, Secher $\mathrm{NH}$, Kehlet H: Monitoring of perioperative fluid administration by individualized goal-directed therapy. Acta Anaesthesiol Scand 2007, 51:331-340.

2. Holte K, Sharrock NE, Kehlet H: Pathophysiology and clinical implications of perioperative fluid excess. Br J Anaesth 2002, 89:622-632.

3. Giglio MT, Marucci M, Testini M, Brienza N: Goal-directed haemodynamic therapy and gastrointestinal complications in major surgery: a metaanalysis of randomized controlled trials. Br J Anaesth 2009, 103:637-646.

4. Meregalli A, Oliveira RP, Friedman G: Occult hypoperfusion is associated with increased mortality in hemodynamically stable, high-risk, surgical patients. Crit Care 2004, 8:R60-R65.

5. Davies SJ, Wilson RJ: Preoperative optimization of the high-risk surgical patient. Br J Anaesth 2004, 93:121-128.

6. Tote SP, Grounds RM: Performing perioperative optimization of the high-risk surgical patient. Br J Anaesth 2006, 97:4-11.

7. Conway DH, Mayall R, bdul-Latif MS, Gilligan S, Tackaberry C: Randomised controlled trial investigating the influence of intravenous fluid titration using oesophageal Doppler monitoring during bowel surgery. Anaesthesia 2002, 57:845-849.

8. Wakeling HG, McFall MR, Jenkins CS, Woods WG, Miles WF, Barclay GR, Fleming SC: Intraoperative oesophageal Doppler guided fluid management shortens postoperative hospital stay after major bowel surgery. Br J Anaesth 2005, 95:634-642.

9. Chytra I, Pradl R, Bosman R, Pelnar P, Kasal E, Zidkova A: Esophageal Doppler-guided fluid management decreases blood lactate levels in multiple-trauma patients: a randomized controlled trial. Crit Care 2007, 11:R24.

10. Gan TJ, Soppitt A, Maroof M, El-Moalem H, Robertson KM, Moretti E, Dwane P, Glass PS: Goal-directed intraoperative fluid administration reduces length of hospital stay after major surgery. Anesthesiology 2002, 97:820-826.

11. Lopes MR, Oliveira MA, Pereira VO, Lemos IP, Auler JO Jr, Michard F: Goaldirected fluid management based on pulse pressure variation monitoring during high-risk surgery: a pilot randomized controlled trial. Crit Care 2007, 11:R100.

12. Pearse R, Dawson D, Fawcett J, Rhodes A, Grounds RM, Bennett ED: Early goal-directed therapy after major surgery reduces complications and duration of hospital stay. A randomised, controlled trial [ISRCTN38797445]. Crit Care 2005, 9:R687-R693.

13. Sinclair $S$, James $S$, Singer M: Intraoperative intravascular volume optimisation and length of hospital stay after repair of proximal femoral fracture: randomised controlled trial. BMJ 1997, 315:909-912.

14. Donati A, Loggi S, Preiser JC, Orsetti G, Munch C, Gabbanelli V, Pelaia P, Pietropaoli P: Goal-directed intraoperative therapy reduces morbidity and length of hospital stay in high-risk surgical patients. Chest 2007, 132:1817-1824.

15. Wilson J, Woods I, Fawcett J, Whall R, Dibb W, Morris C, McManus E: Reducing the risk of major elective surgery: randomised controlled trial of preoperative optimisation of oxygen delivery. BMJ 1999, 318:1099-1103.

16. Venn R, Steele A, Richardson P, Poloniecki J, Grounds M, Newman P: Randomized controlled trial to investigate influence of the fluid challenge on duration of hospital stay and perioperative morbidity in patients with hip fractures. BrJ Anaesth 2002, 88:65-71.

17. Lefrant JY, Bruelle P, Aya AG, Saissi G, Dauzat M, de La Coussaye JE, Eledjam JJ: Training is required to improve the reliability of esophageal Doppler to measure cardiac output in critically ill patients. Intensive Care Med 1998, 24:347-352.

18. Morgan BC, Crawford EW, Hornbein TF, Martin WE, Guntheroth WG: Hemodynamic effects of changes in arterial carbon dioxide tension during intermittent positive pressure ventilation. Anesthesiology 1967, 28:866-873.

19. Reuter DA, Felbinger TW, Kilger E, Schmidt C, Lamm P, Goetz AE: Optimizing fluid therapy in mechanically ventilated patients after cardiac surgery by on-line monitoring of left ventricular stroke volume variations. Comparison with aortic systolic pressure variations. $\mathrm{Br} J$ Anaesth 2002, 88:124-126.

20. Solus-Biguenet $\mathrm{H}$, Fleyfel M, Tavernier B, Kipnis E, Onimus J, Robin E, Lebuffe G, Decoene C, Pruvot FR, Vallet B: Non-invasive prediction of fluid responsiveness during major hepatic surgery. Br J Anaesth 2006 97:808-816.

21. Hofer CK, Senn A, Weibel L, Zollinger A: Assessment of stroke volume variation for prediction of fluid responsiveness using the modified FloTrac and PiCCOplus system. Crit Care 2008, 12:R82.

22. Derichard A, Robin E, Tavernier B, Costecalde M, Fleyfel M, Onimus J, Lebuffe G, Chambon JP, Vallet B: Automated pulse pressure and stroke volume variations from radial artery: evaluation during major abdominal surgery. Br J Anaesth 2009, 103:678-684.

23. Perel A: Automated assessment of fluid responsiveness in mechanically ventilated patients. Anesth Analg 2008, 106:1031-1033.

24. De BD, Heenen S, Piagnerelli M, Koch M, Vincent JL: Pulse pressure variations to predict fluid responsiveness: influence of tidal volume. Intensive Care Med 2005, 31:517-523.

25. Reuter DA, Bayerlein J, Goepfert MS, Weis FC, Kilger E, Lamm P, Goetz AE: Influence of tidal volume on left ventricular stroke volume variation measured by pulse contour analysis in mechanically ventilated patients. Intensive Care Med 2003, 29:476-480.

26. Kubitz JC, Annecke T, Forkl S, Kemming GI, Kronas N, Goetz AE, Reuter DA: Validation of pulse contour derived stroke volume variation during modifications of cardiac afterload. Br J Anaesth 2007, 98:591-597.

27. Manecke GR: Edwards FloTrac sensor and Vigileo monitor: easy, accurate, reliable cardiac output assessment using the arterial pulse wave. Expert Rev Med Devices 2005, 2:523-527.

28. Sander M, Spies CD, Grubitzsch H, Foer A, Muller M, von HC: Comparison of uncalibrated arterial waveform analysis in cardiac surgery patients with thermodilution cardiac output measurements. Crit Care 2006, 10:R164.

29. Mayer J, Boldt J, Schollhorn T, Rohm KD, Mengistu AM, Suttner S: Semiinvasive monitoring of cardiac output by a new device using arterial pressure waveform analysis: a comparison with intermittent pulmonary artery thermodilution in patients undergoing cardiac surgery. Br J Anaesth 2007, 98:176-182.

30. Prasser C, Bele S, Keyl C, Schweiger S, Trabold B, Amann M, Welnhofer J, Wiesenack C: Evaluation of a new arterial pressure-based cardiac output device requiring no external calibration. BMC Anesthesiol 2007, 7:9.

31. Senn A, Button D, Zollinger A, Hofer CK: Assessment of cardiac output changes using a modified FloTrac/Vigileo algorithm in cardiac surgery patients. Crit Care 2009, 13:R32.

32. McGee WT, Horswell JL, Calderon J, Janvier G, Van ST, Van den BG, Kozikowski L: Validation of a continuous, arterial pressure-based cardiac output measurement: a multicenter, prospective clinical trial. Crit Care 2007, 11:R105.

33. Mayer J, Boldt J, Wolf MW, Lang J, Suttner S: Cardiac output derived from arterial pressure waveform analysis in patients undergoing cardiac surgery: validity of a second generation device. Anesth Analg 2008, 106:867-872. table

34. Manecke GR Jr, Auger WR: Cardiac output determination from the arterial pressure wave: clinical testing of a novel algorithm that does not require calibration. J Cardiothorac Vasc Anesth 2007, 21:3-7.

35. Button D, Weibel L, Reuthebuch $O$, Genoni M, Zollinger A, Hofer CK: Clinical evaluation of the FloTrac/Vigileo system and two established continuous cardiac output monitoring devices in patients undergoing cardiac surgery. Br J Anaesth 2007, 99:329-336.

36. Lahner D, Kabon B, Marschalek C, Chiari A, Pestel G, Kaider A, Fleischmann $\mathrm{E}$, Hetz $\mathrm{H}$ : Evaluation of stroke volume variation obtained by arterial pulse contour analysis to predict fluid responsiveness intraoperatively. Br J Anaesth 2009, 103:346-351.

37. Hamm JB, Nguyen BV, Kiss G, Wargnier JP, Jauffroy A, Helaine L, Arvieux CC, Gueret G: Assessment of a cardiac output device using arterial pulse waveform analysis, Vigileo, in cardiac surgery compared to pulmonary arterial thermodilution. Anaesth Intensive Care 2010, 38:295-301

38. Umgelter A, Reindl W, Schmid RM, Huber W: Is supra-ventricular arrhythmia a reason for the bad performance of the FlowTrac device? Crit Care 2007, 11:406. 
39. Copeland GP, Jones D, Walters M: POSSUM: a scoring system for surgical audit. Br J Surg 1991, 78:355-360.

40. Hoste EA, Clermont G, Kersten A, Venkataraman R, Angus DC, De BD, Kellum JA: RIFLE criteria for acute kidney injury are associated with hospital mortality in critically ill patients: a cohort analysis. Crit Care 2006, 10:R73.

41. Garner JS, Jarvis WR, Emori TG, Horan TC, Hughes JM: CDC definitions for nosocomial infections, 1988. Am J Infect Control 1988, 16:128-140.

42. Mayer J, Boldt J, Mengistu A, Rohm KD, Suttner S: Goal-directed intraoperative therapy based on autocalibrated arterial pressure waveform analysis reduces hospital stay in high-risk surgical patients: a randomized, controlled trial. Crit Care 2010, 14:R18.

43. Compton FD, Zukunft B, Hoffmann C, Zidek W, Schaefer JH: Performance of a minimally invasive uncalibrated cardiac output monitoring system (Flotrac/Vigileo) in haemodynamically unstable patients. Br J Anaesth 2008, 100:451-456.

44. Biais M, Nouette-Gaulain K, Cottenceau V, Vallet A, Cochard JF, Revel P, Sztark F: Cardiac output measurement in patients undergoing liver transplantation: pulmonary artery catheter versus uncalibrated arterial pressure waveform analysis. Anesth Analg 2008, 106:1480-1486. table

45. Biancofiore G, Critchley LA, Lee A, Bindi L, Bisa M, Esposito M, Meacci L, Mozzo R, DeSimone P, Urbani L, Filipponi F: Evaluation of an uncalibrated arterial pulse contour cardiac output monitoring system in cirrhotic patients undergoing liver surgery. Br J Anaesth 2009, 102:47-54.

46. Buettner M, Schummer W, Huettemann E, Schenke S, van HN, Sakka SG: Influence of systolic-pressure-variation-guided intraoperative fluid management on organ function and oxygen transport. $\mathrm{Br} J$ Anaesth 2008, 101:194-199.

47. Kubitz JC, ForkI S, Annecke T, Kronas N, Goetz AE, Reuter DA: Systolic pressure variation and pulse pressure variation during modifications of arterial pressure. Intensive Care Med 2008, 34:1520-1524.

48. Pearse R, Dawson D, Fawcett J, Rhodes A, Grounds RM, Bennett ED: Changes in central venous saturation after major surgery, and association with outcome. Crit Care 2005, 9:R694-R699.

49. Multicentre study on peri- and postoperative central venous oxygen saturation in high-risk surgical patients. Crit Care 2006, 10:R158.

doi: $10.1186 /$ cc 9070

Cite this article as: Benes et al., Intraoperative fluid optimization using stroke volume variation in high risk surgical patients: results of prospective randomized study Critical Care 2010, 14:R118

\section{Submit your next manuscript to BioMed Central} and take full advantage of:

- Convenient online submission

- Thorough peer review

- No space constraints or color figure charges

- Immediate publication on acceptance

- Inclusion in PubMed, CAS, Scopus and Google Scholar

- Research which is freely available for redistribution

Submit your manuscript at www.biomedcentral.com/submit
C Biomed Central 